${ }^{1}$ Department of Child Dental Health, Lagos State University Teaching Hospital, Lagos, Nigeria.

${ }^{2}$ Department of Child Dental Health, College of Medicine, University of Lagos, Lagos, Nigeria.

${ }^{3}$ Department of Preventive Dentistry, Faculty of Dentistry, University of Medical Sciences, Ondo State, Nigeria.

${ }^{4}$ Department of Preventive Dentistry, Faculty of Dentistry, Lagos State University College of Medicine, Lagos, Nigeria.

${ }^{5}$ Faculty of Family Dentistry, National Postgraduate Medical College of Nigeria, Lagos, Nigeria.

${ }^{6}$ Department of Child Dental Health, Faculty of Dentistry, Lagos State University College of Medicine, Lagos, Nigeria.

Corresponding author: Dr Afolabi Oyapero Senior Lecturer, Department of Preventive Dentistry Lagos State University College of Medicine, Lagos, Nigeria.

Email:fola_ba@yahoo.com

Editor: Dr Altair A. Del Bel Cury

Received: July 14, 2021

Accepted: September 6, 2021

\section{Randomized controlled trial on the effectiveness of silver diamine fluoride in arresting caries in Lagos, Nigeria}

\author{
Moses Okechukwu Azuoru ${ }^{1}$ (D), Modupe Olufunmilayo \\ Ashiwaju $^{2}$ (D) Augustine Edomwonyi ${ }^{3}$ (D), Afolabi \\ Oyapero $^{4}$ (D) Bola Obisesan ${ }^{5}$ (D), Aderinsola Omotuyole ${ }^{6}$ (D)
}

Treatment of dental caries in children still remains challenging due to lack of cooperation with conventional treatment modalities. Recently, the use of Silver Diamine Fluoride (SDF) has proved useful in addressing this challenge. Aim: This clinical trial aimed to evaluate the effectiveness of Silver Diamine Fluoride (SDF) in arresting caries in children in Lagos, Nigeria. Methods: This was a phase III balanced randomized controlled school based interventional study on 240 children. The study group was treated with SDF while GIC was used in the control group. Follow up visits in 2 weeks, 1 month, and 3 months were carried out to assess the treatment outcome. Inferential statistics with the use of Pearson Chi-square test and Independent Student t-test were used at 5\% level of significance. Results: There was significant relationship between SDF and caries arrest in 2 weeks, 1 month and 3 months' assessment period ( $p=0.001$ ). The control group showed continuous decline $(71.7 \%, 54.3 \%$ and $50.9 \%)$ in restorative success from 2 weeks to 3 months respectively. The mean \pm SD and Confidence Interval $(\mathrm{Cl})$ of arrested caries in the SDF group were $113 \pm 1.24$ and $113.1-113.5$ respectively. In the control group the mean $\pm \mathrm{SD}$ and $\mathrm{Cl}$ of restorative success were $69.3 \pm 11.8$ and $67.2-71.4$. The effect size was 5.24. Conclusion: The result of the study showed that SDF was effective in arresting caries in children without any harm and there was statistically significant difference in the use of $38 \%$ SDF in arresting caries in children.

Keywords: Dental caries. Glass ionomer cement. Fluorides, topical. 


\section{Introduction}

According to the Global Burden of Disease Study, an estimated 3.8 billion people are affected by dental caries ${ }^{1}$. In Nigeria, the prevalence of caries among children is still high despite current preventive and control strategies ${ }^{2,3}$. Recently, Adeniyi et al. ${ }^{4}$ reported $14.8 \%$ prevalence of dental caries among children aged 5 - 10 years in Lagos, Nigeria. Though this figure is still within the WHO Millennium Development Goals target for dental caries, its impacts on children's health cannot be over emphasized as it is estimated to be the most prevalent chronic childhood disease worldwide $^{5}$. These impacts include pain and discomfort, difficulty in masticating, sleep and speech disturbance, poor self-esteem and social isolation among others. It can also negatively affect body weight, growth, school attendance, and school performance if left untreated ${ }^{5}$. The commonest treatment protocol for dental caries is traditional surgical intervention but prevention of caries is more cost effective and less invasive ${ }^{6}$. With the recent change from the surgical model, which places emphasis on restorative treatment, to a medical model which focuses on disease prevention and conservation of tooth structure, caries management in the present decade is fast becoming more patient friendly, effective and efficient?

Despite recent advances, the management of dental caries still remains challenging particularly with children, the aged, vulnerable populations and special health care needs patients, where gaining cooperation is still a significant problem in traditional restorative treatment of dental caries $^{8}$. Furthermore, among those from low economic class, access to care and cost are hindrances to conventional dental caries treatment ${ }^{8,9}$. Thus, innovative treatment approach that reduces the burden of care on patients and the need for more comfortable, effective and efficient treatment protocols that would promote patient cooperation have continued to engage dental researchers and clinicians in recent times. The primary focus in these researches has been the use of chemotherapeutic agents in the prevention and arrest of caries particularly in children ${ }^{10}$. Consequently, a variety of chemotherapeutic agents such as metal ions, antibiotics and various types of fluoride containing agents have been developed, tested and used for preventing and arresting caries ${ }^{11}$. A notable innovative therapy among these is the use of Silver Diamine Fluoride (SDF) ${ }^{10}$.

Silver Diamine Fluoride has clinical usefulness in children when patient cooperation for restorative dentistry is difficult due to situational anxiety, young age or intellectual and developmental disabilities ${ }^{12,13}$. SDF is also very useful where the restoration of primary tooth that is about to exfoliate is not an option. Since this process requires non-invasive procedures, the risk of cross-infection is significantly reduced. Clinical studies have shown that SDF prevents and arrests caries in children ${ }^{13}$. A review on SDF concluded that it is a safe, effective and efficient caries control agent that can be employed to meet the WHO Millennium Development Goals for 21 st-Century ${ }^{12-15}$. Randomized clinical trials on the effectiveness of SDF have been carried out in United States, Europe and Asia ${ }^{15-17}$. In an ex-vivo study, Mei et al. ${ }^{18}$ reported a highly remineralized rich zone in calcium and phosphate on arrested caries lesion of primary teeth with SDF application. In a related study, Milgrom et al. ${ }^{19}$ also reported that application of $38 \%$ SDF arrested caries and was effective for the short-term treatment of caries 
in pre-school age children. There is however no available published or unpublished research in Nigeria nor Africa on the effectiveness of SDF in arresting caries after meticulous search of relevant literature. There is also no research evidence to support the use of SDF in Africa despite the growing interest in this agent. Thus, the aim of this study was to evaluate the effectiveness of 38\% Silver Diamine Fluoride in arresting caries in children in Lagos, Nigeria.

\section{Materials and methods}

Description of study area: The study was conducted in Lagos, Nigeria from October 2019 to March 2020. Lagos lies between latitude 6.465422 and longitude $3.406448^{20}$. It is bordered essentially in the south by Atlantic Ocean and hence it is a coastal city. As epicenter of commerce and industry, it is a heterogeneous State with mixed proportion of different ethno-religious groups and socioeconomic class. More significantly, because it is a commercial city the production and consumption of refined sugars is very high ${ }^{21}$. This is a favorable predisposing factor for the development of caries.

Study population: Children aged 3 - 10 years living in Lagos, Nigeria made up the study population. According to the National Population Commission the population of children aged 0 - 9 years in Lagos State in 2006 population census was 2,109,8622. The study age group ranks highest in the prevalence of dental caries in Lagos State estimated at $14.8 \%{ }^{4}$. It is also the group with high incidence of Early Childhood Caries $^{4}$. More importantly, it is the age group that poses difficulty in tolerating traditional restorative technique due to situational anxiety, low emotional stability and low intellectual understanding ${ }^{23}$.

Study design: The study design was a Phase III Balanced Randomized Controlled Trial; Parallel Groups, Multicentre School Based Interventional Study conducted in Lagos Nigeria.

Ethical considerations: Approval for the study was obtained from the Lagos State University Teaching Hospital (LASUTH) Health Research and Ethics Committee with reference number LREC/06/10/1221 dated 31 July 2019. Approval for the study was also obtained from the Lagos State Government with reference no: LS/C-530/T.3/755-756 dated 28 Oct 19 with respect to the use of schools and primary school children for the study. Approval was also obtained from various school authorities where the study was conducted through the Education Secretaries of various districts used for the study. Informed and signed consents were obtained from parents, guardians and caregivers of children who participated in the study. Informed assent was obtained from the children during the study. Participants with increased caries activities during the course of the study were planned to be withdrawn from the study and given conventional treatment.

Registration of trial: The clinical trial was registered at Pan African Clinical Trials Registry (PACTR) with Trial no: PACTR201908699150281 dated 16/04/2019. Trial was registered in accordance with WHO and ICMJE standards.

Sampling Technique: A multistage sampling technique was utilized. Eight (8) public primary schools in Lagos State were selected in the first stage using computer gen- 
erated random numbers, with the list of schools serving as the sampling frame. The second stage involved selection of classes in eligible classes in the schools by simple random sampling using the nominal rolls as a sampling frame. Children who fulfilled the eligibility criteria for the study were eventually enlisted. Eventual allocation of the recruited sample population into 2 equal groups was also done by simple randomization.

Sample size estimation: This was done with the formula:

$\mathrm{n}=\frac{\left(Z_{a}+Z_{\beta}\right)^{2} \times 2 p(1-p)}{d^{2}}$ where:

$\mathrm{n}=$ sample size required in each group; $\mathrm{Z}_{\mathrm{a}}=$ value for $\mathrm{a}$ at desired confidence level of $95 \% ; a=0.05$ (two-tailed test) $=1.96 ; Z_{\beta}=$ value of $\beta$ error, which is $1-\beta$ (statistical power). At statistical power of $95 \%$ and $\beta$ error of $5 \%, z_{\beta}=1.94 ; p=$ proportion of dental caries in children aged $5-10$ years in a reference study (prevalence is $14.8 \% ; 0.148)^{4}$; and $d=$ the minimum difference in the clinical performance between the two study groups. The minimum difference for this study was set at $20 \%$ in order to increase power of the study and give more validity to the study. Hence $d=20 \%$.

Thus, $\mathrm{n}=96.7$

With $10 \%$ attrition or follow up losses (9.67), minimum sample for each group was 107 or 214 for the two groups. However, 240 subjects were recruited in all.

\section{Eligibility Criteria}

Inclusive Criteria: Children aged 3 -10 years, with dental caries ICDAS 5 or 6 , with signed consent form from parents/guardians and signed or thumb printed the assent form that allowed for oral examination and application of intervention medicament and control treatment were included.

Exclusion Criteria: Children with symptomatic carious tooth (toothache or sensitivity), mobile carious tooth, with dental caries ICDAS 0-4; those who were exposed to fluoride from other sources apart from a dentifrice; those that were allergic to silver or heavy metals; with Amelogenesis or Dentinogenesis Imperfecta; with oral ulceration, stomatitis, swelling or abscess; with cooperation challenge; obviously mentally retarded; systemic illness like Asthma, Epilepsy, Leukaemia, Kidney disease and those whose parent or guardian could not understand the consent documentation were excluded from the study.

Research Questionnaire: An interviewer administered close ended structured questionnaire developed from previously validated questionnaires was used. It comprised sections A to E. Section A to D captured a participant's biodata, history, examination, and intervention and control data while section $\mathrm{E}$ documented the study outcomes.

Dental Caries Examination Tools and Treatment Set: The caries examination tools and treatment set comprised a comfortable field examination mobile dental chair unit which was set up in a well-lit and airy room in the schools used for the study. The examination instrument comprised standard WHO periodontal probe, wooden spatula, mouth mirror, $5 \mathrm{ml}$ hypodermic syringes with water, cotton gauze and rolls, meth- 
ylated spirit and dental tray. The treatment set for SDF comprised dispensing dish, microbrushes, guage, cotton wool/rolls and dental tray. The treatment set for GIC restoration comprised atraumatic hand instruments (excavators, condensers, explorer, tweezers, dental hatchet, mouth mirrors, carvers, examination probes and periodontal probes), mixing pads, mixing spatula, plastic applicators, gauge, wedges, cotton wool/rolls/pellets, petroleum jelly and dental tray. The researchers were trained and calibrated to use these tools and instrument for diagnosis of ICDAS 5 or $6^{24}$, treatment of caries and assessing the study outcome which is Arrested Caries using ICDAS II criteria by a Consultant Paediatric Dentist.

Intervention Medicament and Control Medicament: The intervention or experimental medicament for the study was 38\% Silver Diamine Fluoride: TEDEQUIM S.R.L B. ${ }^{25}$ The control Medicament was Glass Ionomer Cement (GIC): PrevestDenPro. ${ }^{26}$

\section{Details and Sequence of Data Collection}

\section{Phase I - Recruitment and Intervention Phase.}

Day 1: On the first day in each school, school children aged 3- 10 which cut across Nursery 1-2 and Primary 1-6 were examined for dental caries ICDAS 5 and 6 . The examination was done in a well-lit open hall or school clinic provided by the school. The children were made to sit comfortably in a mobile dental chair unit. Examination of ICDAS 5 and 6 caries was done by direct visual examination using wooden spatula by two calibrated examiners. Records of the children with ICDAS 5 and 6 were taken and documented. The documented children with ICDAS 5 and 6 were given consent document and forms to give to their parents to authorize intervention.

Day 2 - Interventions: The children that were given the consent document and forms were recalled on day 2 . The children with signed consent forms were randomized into experimental group and control group by randomization. The children in the two groups were counseled and then signed or thumb print the assent form. The children in Group 1 received SDF while the children in Group 2 received GIC.

Administration of SDF: The children in Group 1 thereafter were treated with topical SDF. Using a comfortable mobile chair unit, Section A - C of the Questionnaire were administered to the children by the investigators. Thereafter, the affected carious tooth with ICDAS 5 or 6 was gently cleaned, dried and isolated with cotton rolls. Two drops of SDF solution was then applied with a disposable microbrush on the carious tooth. The treated participants were instructed not to rinse for the next 10 minutes. Subjects were also advised to continue their normal oral hygiene care. The intervention received with the treatment date as well as the tooth treated (ICDAS 5 or 6 ) and the caries class was all documented on Section D (Intervention Section) of the questionnaire. The date of the treatment and the school name were also recorded in the study book to calculate subsequent time of follow up visits.

Administration of GIC: The children in Group 2 received GIC intervention. Like in Group 1, the children in Group 2 were administered Section A - C of the Questionnaire by the investigator and the research assistant (House Officer). Thereafter the researcher administered conventional GIC. The carious lesion of the affected tooth 
with ICDAS 5 or 6 was excavated with disposable plastic excavator. The tooth was cleaned, dried and isolated with cotton rolls. Conventional GIC was then mixed in a high viscosity consistency and condensed on the tooth cavity and allowed to set. Petroleum jelly as a separating medium was then used to seal the GIC surface from saliva. The participants were instructed not to rinse for the next 10 minutes. Participants were also advised to continue their normal oral hygiene care. The intervention received with the treatment date as well as the tooth treated (ICDAS 5 or 6 ) and the caries class was all documented on Section D (Intervention Section) of the questionnaire. The dates of the treatment intervention and the school name were also recorded in the study book to calculate subsequent time of follow up visits.

Phase II - Outcome Phase: Since it was a prospective study the second phase assessed the treatment outcomes. The outcomes were assessed in 2 weeks, 1 month and 3 months respectively. The outcomes that were assessed were as follows:

Primary Outcome: The expected primary outcome for the experimental group was arrested caries while that for the control group was restorative success.

Arrested Caries for SDF Group (Experimental Group): Arrested caries was assessed using the ICDAS || criteria and findings were recorded in the questionnaire. The researcher examined the treated teeth for arrested and active caries. The assessment was done using visual tactile examination with aid of WHO probe and mouth mirror using the ICDAS II criteria ${ }^{24}$ to classify active and arrested caries.

Restorative Success for GIC Group (Control Group): The restorative success of Glass Ionomer Cement was determined when signs of restorative failure were absent. Signs of GIC restorative failure included restoration losses (full or partial loss), fractures and wear. Assessment of restorative failure was done using both visual and tactile examination with the aid of examination probe and mouth mirror. The restoration was gently probed to assess for any sign of failure such as fracture, wear, partial loss or total loss. Findings were recorded accordingly in the participant's questionnaire. The type of restorative failure was also noted in each case. The follow-up outcome assessments were carried out in 2 weeks, 1 month and 3 months respectively after the intervention.

Secondary Outcomes: The secondary outcomes that were assessed in both the experimental group and control group included toothache, sensitivity, stain, nausea, vomiting, silver allergy, oral soft tissue ulceration, rashes and any other noted complications. The assessments for secondary outcomes were done concurrently with the assessment of primary outcome. There was no case that necessitated hospital referral.

Data analysis: The obtained data was analyzed using Statistical Package for Social Sciences (SPSS IBM New York, USA) Windows Version 23. Data were coded and entered into Microsoft Excel spreadsheet and later imported into SPSS for cleaning and analysis. Descriptive statistics with the use of frequencies, percentage/proportion, mean and standard deviation were used to summarize data. Inferential statistics with the use of Pearson Chi-square test, Fisher exact test and Independent Student t-test were used to test for association between bivariate at $5 \%$ level of significance (95\% Confidence interval). A value of $\mathrm{P}<0.05$ was considered statistically significant. 


\section{Results}

A total of 240 school children aged $4-10$ years participated in the study. Out of these, 124(51.7\%) were male while 116(48.3\%) were female, giving a male-to-female ratio of 1.1:1. The SDF group had 64(53.3\%) males and 56(46.7\%) females giving male to female ratio of 1.1:1 The Control group had 60 (50.0\%) male and 60(50.0\%) female giving male to female ratio of 1:1.

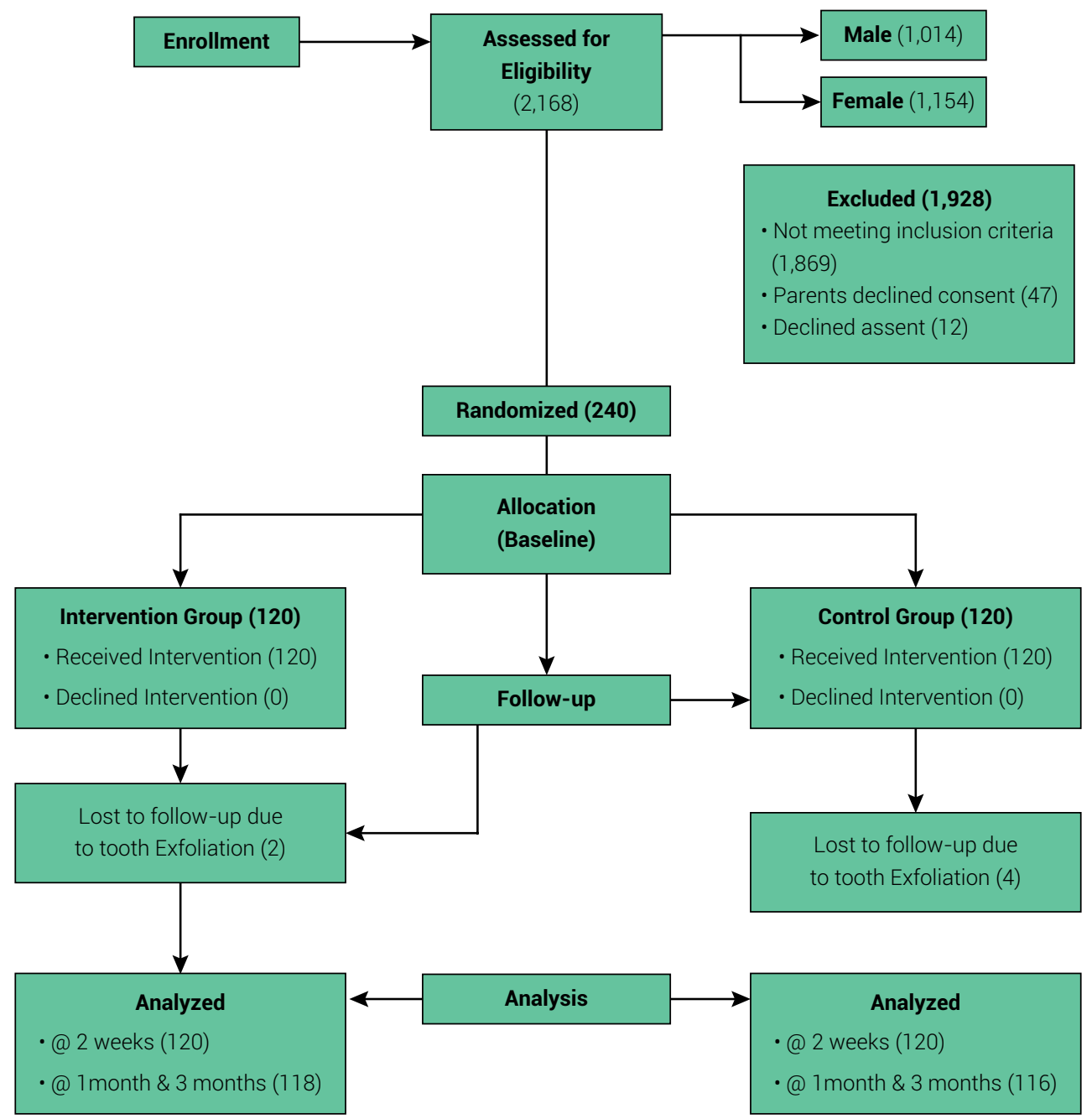

Figure 1. Participants Flowchart

The mean dmft in SDF group was $1.87 \pm 0.9$ while the mean of control group was $1.75 \pm 0.9$. The total mean dmft was $1.81 \pm 0.9$. Overall, dmft score 1 had the highest percentage of $47.9 \%$ closely followed by DMFT score 2 with $32.8 \%$. dmft score 5 had the lowest percentage of $0.04 \%$. There was no significant difference between the $\mathrm{dmft}$ scores in the two groups ( $p>0.05)$. - Table 1 
Table 1. Distribution of dmft scores of participants

\begin{tabular}{|c|c|c|c|c|c|}
\hline Variables & $\begin{array}{l}\text { SDF Group } \\
(\mathrm{n}=120) \\
\mathrm{n}(\%)\end{array}$ & $\begin{array}{c}\text { Control } \\
(n=120) \\
n(\%)\end{array}$ & $\begin{array}{c}\text { Total } \\
(\mathrm{n}=240) \\
\mathrm{n}(\%)\end{array}$ & t-test* & $\mathrm{p}$ \\
\hline \multicolumn{6}{|c|}{ dmft Scores } \\
\hline 1 & $52(43.3)$ & $63(52.5)$ & $115(47.9)$ & 1.032 & 0.302 \\
\hline 2 & $42(35.0)$ & $35(29.2)$ & $77(32.8)$ & & \\
\hline 3 & $17(14.2)$ & $14(11.6)$ & $31(12.9)$ & & \\
\hline 4 & $8(6.6)$ & $6(5.0)$ & $14(5.8)$ & & \\
\hline 5 & $0(0.0)$ & $1(0.8)$ & $1(0.04)$ & & \\
\hline 6 & $1(0.8)$ & $1(0.8)$ & $2(0.1)$ & & \\
\hline Mean \pm SD & $1.87 \pm 0.9$ & $1.75 \pm 0.9$ & $1.81 \pm 0.9$ & & \\
\hline
\end{tabular}

*Independent t-test

Table 2 shows the arrested/restorative success at different intervals of assessment. At 2 weeks' outcome assessment, 94.2\% of the treated carious teeth in the SDF group showed hard arrested dentine, while $71.7 \%$ of the restored carious teeth in the control group showed restorative success. At the 1-month outcome assessment, the SDF group had $97.5 \%$ of the teeth showing arrested caries, while the control group had $54.3 \%$ showing restorative success. At 3 months' outcome assessment, $94.9 \%$ of the carious lesions in teeth treated with SDF were still arrested, while the control group showed $50.9 \%$ of teeth with restorative success. Chi-square test of independence showed there was significant relationship between SDF and caries arrest at 2 weeks, 1 month and 3 months' assessment period respectively $(p=0.001)$. There was a marginal increase of $3.3 \%$ in caries arrest in the SDF group between 2 weeks and 1-month outcome assessment. However, there was a slight decline in caries arrest of $2.6 \%$ between 1 month and 3 months of outcome assessment. The control group showed continuous decline $(71.7 \%, 54.3 \%$ and $50.9 \%)$ in restorative success from 2 weeks to 3 months respectively.

Table 2. Distribution of Arrested Caries /Restorative Success at different intervals of assessment

\begin{tabular}{|c|c|c|c|c|c|}
\hline Variables & $\begin{array}{c}\text { SDF group } \\
(n=120) \\
n(\%)\end{array}$ & $\begin{array}{c}\text { Control } \\
(n=120) \\
n(\%)\end{array}$ & Total & $x^{2}$ & $\mathrm{p}$-value \\
\hline \multicolumn{6}{|c|}{2 weeks' assessment } \\
\hline Success & $113(94.2)$ & $86(71.7)$ & 199(82.9) & 21.444 & $<0.001$ * \\
\hline Failure & $7(5.8)$ & $34(238.3)$ & $41(17.1)$ & & \\
\hline \multicolumn{6}{|c|}{1 month's assessment } \\
\hline Success & 115(97.5) & $63(54.3)$ & 178(79.1) & $60.031^{\star \star}$ & $<0.001$ * \\
\hline Failure & $3(2.5)$ & $53(45.7)$ & $56(23.9)$ & & \\
\hline \multicolumn{6}{|c|}{3 months' assessment } \\
\hline Success & 112(94.9) & $59(50.9)$ & $171(73.1)$ & 57.700 & $<0.001$ * \\
\hline Failure & $6(5.1)$ & $57(49.1)$ & $63(26.9)$ & & \\
\hline
\end{tabular}

Two (2) and 4 subjects lost to follow up at experimental group and control group respectively for one month and 3 months' assessments

**Fischer exact test 
Table 3 shows the intervention failure at different intervals of assessment. In the SDF group, observed failure signs ranged from presence of dull enamel/dentine to soft dentine on examination. In the control group observed signs of failure ranged from partial loss of restoration to total loss and wear/fracture of restoration. At 2 weeks' outcome assessment, there were 7 non arrested carious teeth in the SDF group: $3(42.9 \%)$ were as a result of soft dentine while $4(57.1 \%)$ were as a result of dull enamel/dentine. At 1-month assessment, SDF group showed 3 non arrested cavities evidenced by dull enamel/ dentine. At three (3) months assessment. 5 treated teeth had dull dentine while one (1) had soft dentine. The control group recorded a greater number of restorative failures. There was a progressive failure rate of restoration. The restorative failure rate increased with time of assessment. A total of 57 restorative failures were observed out of 120 restored teeth giving a restorative failure rate of $47.5 \%$. At 2 weeks' assessment there were a total of 34 restorative failures; $13(38.2 \%)$ due to partial loss of restoration, $15(44.1 \%)$ due to total loss and $6(17.6 \%)$ due to wear of restoration. One-month assessment showed a total of 50 restorative failures; $9(16.1 \%)$ was due to partial loss, $40(75.5 \%)$ as a result of total loss and one (1.9\%) due to wear of restoration. At 3 months' assessment, there were 57 restorative failures: $7(12.3 \%)$ due to partial loss, $49(86.0 \%)$ as a result of total loss and one (1.8\%) due to wear of restoration.

Table 3. Distribution of intervention failure at different intervals of assessment

\begin{tabular}{|c|c|c|c|c|c|}
\hline & $\begin{array}{c}\text { SDF group } \\
\mathrm{n}(\%)\end{array}$ & $\begin{array}{c}\text { Control } \\
\mathrm{n}(\%)\end{array}$ & Total & $\mathrm{X}^{2}$ & $\mathrm{p}$-value \\
\hline 2 weeks & & & & 41.000 & $<0.001$ * \\
\hline Dull enamel/Dentine & $4(57.1)$ & $0(0.0)$ & $4(9.8)$ & & \\
\hline Partial loss of filling & $0(0.0)$ & 13(38.2) & $17(31.7)$ & & \\
\hline Soft dentine & $3(42.9)$ & $0(0.0)$ & $3(7.3)$ & & \\
\hline Total loss of filling & $0(0.0)$ & $15(44.1)$ & $15(36.6)$ & & \\
\hline Wear of filling & $0(0.0)$ & $6(17.6)$ & $6(14.6)$ & & \\
\hline 1 month & & & & 26.415 & $<0.001$ * \\
\hline Dull enamel/Dentine & $3(100.0)$ & $3(5.7)$ & $6(10.7)$ & & \\
\hline Partial loss of filling & $0(0.0)$ & $9(17.0)$ & $9(16.1)$ & & \\
\hline Total loss of filling & $0(0.0)$ & $40(75.5)$ & $40(71.4)$ & & \\
\hline Wear of filling & $0(0.0)$ & $1(1.9)$ & $1(1.8)$ & & \\
\hline 3 months & & & & 63.000 & $<0.001$ * \\
\hline Dull enamel/Dentine & $5(83.3)$ & $0(0.0)$ & $5(7.9)$ & & \\
\hline Partial loss of filling & $0(0.0)$ & $7(12.3)$ & $7(11.1)$ & & \\
\hline Soft dentine & $1(16.7)$ & $0(0.0)$ & $1(1.6)$ & & \\
\hline Total loss of filling & $0(0.0)$ & $49(86.0)$ & $49(77.8)$ & & \\
\hline Wear of restoration & $0(0.0$ & $1(1.8)$ & $1(1.6)$ & & \\
\hline
\end{tabular}

*Fisher exact test 
The mean \pm SD and Confidence Interval $(\mathrm{Cl})$ of arrested caries in the SDF group were $113 \pm 1.24$ and $113.1-113.5$ respectively. In the control group the mean \pm SD and $\mathrm{Cl}$ of restorative success were $69.3 \pm 11.8$ and $67.2-71.4$. The effect size ' $d$ ' of the study was 5.24. - Table 4

Table 4. Mean outcome of the two groups at different intervals of assessment and the effect size

\begin{tabular}{lccccc}
\hline Variables & $\begin{array}{c}\text { SDF } \\
\mathbf{n}=120\end{array}$ & $\begin{array}{c}\text { Control } \\
\mathbf{n}=120\end{array}$ & $\begin{array}{c}\text { Mean } \pm \text { SD } \\
\text { SDF }\end{array}$ & $\begin{array}{c}\text { Mean } \pm \text { SD } \\
\text { Control }\end{array}$ & $\mathbf{d}$ \\
\hline Arrested/RS & & & & \\
\hline 2 weeks & 113 & 86 & $113.3 \pm 1.24$ & $69.3 \pm 11.8$ & 5.24 \\
\hline 1 Month & 115 & 63 & & \\
\hline 3 Months & 112 & 59 & $1.5^{*}$ & $141.5^{*}$ \\
\hline Variance & & \multicolumn{5}{c}{$113.1-113.5$} & $67.2-71.4^{* *}$ \\
\hline $95 \% \mathrm{Cl}$ &
\end{tabular}

$\mathrm{d}=$ effect size, ${ }^{*}$ variance, ${ }^{\star *} \mathrm{Cl}$ RS Restorative Success

At two weeks' secondary outcome assessment, $6.7 \%$ of participants reported slight tooth sensitivity in the SDF group while $5.0 \%$ of participants reported tooth sensitivity in the control group. At 1-month, tooth sensitivity reduced in the SDF group to $0.8 \%$ while there was an increase in the control group to $5.2 \%$. At 3 months the percentages of tooth sensitivity remained same for both the SDF group and control group at $0.8 \%$ and $5.2 \%$ respectively. There was significant relationship between tooth sensitivity and treatment modality at 1 month and 3 months' assessment periods $(p<0.05)$. -Table 5

Table 5. Tooth Sensitivity at different intervals of assessment

\begin{tabular}{|c|c|c|c|c|c|}
\hline & $\begin{array}{l}\text { Study group } \\
(n=120)\end{array}$ & $\begin{array}{l}\text { Control } \\
(n=120)\end{array}$ & Total & $\mathrm{X}^{2}$ & $\mathrm{p}$-value \\
\hline \multicolumn{6}{|c|}{2 weeks' assessment } \\
\hline Yes & $8(6.7)$ & $6(5.0)$ & $14(5.8)$ & 0.303 & 0.582 \\
\hline No & 112(93.3) & $114(95.0)$ & $226(94.2)$ & & \\
\hline \multicolumn{6}{|c|}{ 1-month assessment } \\
\hline Yes & $1(0.8)$ & $6(5.2)$ & $7(3.0)$ & $3.770^{\star \star}$ & $0.041 *$ \\
\hline No & 117(99.2) & $110(94.8)$ & $227(97.0)$ & & \\
\hline \multicolumn{6}{|c|}{3 months' assessment } \\
\hline Yes & $1(0.8)$ & $6(5.2)$ & $7(3.0)$ & $3.770 * \star$ & $0.041 *$ \\
\hline No & 117(99.2) & $110(94.8)$ & $227(97.0)$ & & \\
\hline
\end{tabular}

Two (2) and 4 subjects lost to follow up at experimental group and control group for one and 3 months' assessments

**Fischer exact test 
Twenty-Four (24) hours after the interventions, there was no adverse effect in both the SDF group and control group. At 2 weeks follow up $99.2 \%$ of the participants in the SDF group had black stain without pain on the treated tooth while only one (0.8\%) participant reported slight pain in the control group. At 1 month, 98.3\% of the treated teeth in the SDF group showed black stain while none in the control group had any adverse effect. At 3 months follow up the black stain on treated teeth declined to $97.4 \%$ while there was no adverse effect in the control group. No allergic reaction or any other adverse effects was observed in both SDF group and control group at different intervals of assessment. Black teeth stains in the SDF group were statistically significant at 2 weeks, 1 month and 3 months' assessment period $(P<0.001)$. -Table 6

Table 6. Distribution of Adverse effect of intervention at different intervals of assessment

\begin{tabular}{|c|c|c|c|c|c|}
\hline & $\begin{array}{c}\text { SDF group } \\
(n=120) \\
(\%)\end{array}$ & $\begin{array}{c}\text { Control } \\
(n=120) \\
(\%)\end{array}$ & Total & $\begin{array}{l}\text { Fischer } \\
\text { exacts }\end{array}$ & $\mathrm{p}$-value \\
\hline \multicolumn{6}{|l|}{ 24-hour assessment } \\
\hline Nil adverse effect & $120(100.0)$ & $120(100.0)$ & $240(100.0)$ & 0.000 & 1.000 \\
\hline \multicolumn{6}{|l|}{2 weeks' assessment } \\
\hline Black stain & 119(99.2) & $0(0.0)$ & $119(49.6)$ & & \\
\hline Slight pain & $0(0.0)$ & $1(0.8)$ & $1(0.4)$ & 236.033 & $<0.001$ * \\
\hline Nil Black stain/Pain & $1(0.8)$ & $119(99.2)$ & $120(50.0)$ & & \\
\hline \multicolumn{6}{|l|}{ 1-month assessment } \\
\hline Black stain & 116(98.3) & $0(0.0)$ & $116(48.3)$ & 226.802 & $<0.001$ * \\
\hline Nil Black stain/Pain & $2(0.02)$ & $116(100)$ & $118(49.2)$ & & \\
\hline \multicolumn{6}{|l|}{ 3-month assessment } \\
\hline Black stain & $115(97.4)$ & $0(0.0)$ & $116(48.3)$ & 226.802 & $<0.001$ * \\
\hline Nil Black stain/Pain & $3(0.06)$ & $116(100)$ & $118(49.2)$ & & \\
\hline
\end{tabular}

Two (2) and 4 subjects lost to follow up at experimental group and control group respectively for one and 3 months' assessments

\section{DISCUSSION}

The intervention teeth used in the study were posterior primary teeth. Most studies on the effectiveness of SDF in arresting caries in children also used posterior primary molars $23,27,28$. In this study, the second primary molars constituted the highest percentage (57.1\%) of teeth used while the central incisors were least $(0.4 \%)$. The high involvement of primary molars especially the second molar in caries formation could be due to the fact they are big, have broad surface areas with pits, grooves and fissures and are regularly used for chewing. Secondly, within the age bracket of the study, the primary molars experience more time exposure to caries than even the first permanent molars. With respect to ICDAS class, this study observed high ICDAS 5 - ICDAS 6 ratio of 1.5:1. This ratio is in congruence with Rosenblatt et al. ${ }^{14}$ 
and Zhi et al. ${ }^{17}$ in their separate studies using the ICDAS system on the effectiveness of SDF in arresting caries in children. ICDAS 5 caries was more in this study because cavitation is minimal compared to ICDAS 6 and most often asymptomatic. Hence, it is unnoticed or ignored.

The clinical effectiveness of 38\% SDF in arresting dental caries among children has been extensively researched and well documented 17,14,16,29-35. Both RCTs and systematic reviews have been carried out ${ }^{16,29,32-40}$ to evaluate its short and long-term effectiveness. In this study, the caries-arresting rate of $38 \%$ SDF was found to be $94.2 \%$ in 2 weeks, $97.5 \%$ in 1 month and $94.9 \%$ in 3 months. These findings agree comparably with recent clinical trials. Santos $\mathrm{Jr}$ et al. ${ }^{37}$ reported $81 \%$ caries arrest in 1 week and $72.7 \%$ in 5 months. In a short-term clinical trial, Milgrom et al. ${ }^{19}$ demonstrated $77.6 \%$ caries arrest in 2 weeks with 38\% SDF while Clemens et al. ${ }^{32}$ reported $100 \%$ caries arrest after 3 months. Furthermore, Zhi et al. ${ }^{17}$ reported $91 \%$ caries arrest in 6 months and $79 \%$ arrest in 12 months while Llorda et al. ${ }^{33}$ reported $77 \%$ caries arrest in 6 months with 38\% SDF. A systematic review conducted on 8 studies concluded an overall proportion of $81 \%$ caries arrest after SDF treatment ${ }^{38}$ while another systematic review in 2016 documented a 65\% caries arrest with SDF ${ }^{29}$ Findings from this present study are in agreement with the reports from these systematic reviews of the effectiveness of SDF. Both this present study and all the compared recent studies adhered strictly to CONSORT guidelines for conducting RCT; had similar methodology, though the different brands of 38\% SDF used could be responsible for the varying rates of success observed. On the other hand, no recent study has reported low effectiveness. Therefore, this study further validates the effectiveness of SDF in arresting caries in children.

In addition, this study compared the effectiveness of SDF and restorative success rate of ART (GIC) at different intervals in the treatment of caries. Compared to the restorative success in the control group, SDF with statistically significant higher rates of treatment success at 2 weeks, 1 month and 3 months' treatment intervals over the control $(p<0.001)$. This also is in line with the reports of studies that compared the effectiveness of SDF and GIC at different intervals. Zhi et al. ${ }^{17}$, Monse et al. ${ }^{39}$, Dos Santos et al. ${ }^{40}$ and Braga et al. ${ }^{41}$ who all reported statistically significantly higher success rates $(p<0.05)$. Additionally, in order to appreciate the clinical importance of SDF in arresting caries, the effect size was also evaluated. The effect size of this study was $5.24(p<0.001)$. Most studies on SDF failed to report effect size. However, Castillo et al. ${ }^{42}$ in a study comparing the effectiveness of SDF and placebo in arresting caries reported a higher effect size of 12.4 compared to this study. The difference in effect size could be due to the fact that higher effect size is expected to be produced with placebo as against an active treatment. Nonetheless, this high effect size underlines the invaluable clinical importance of SDF in arresting caries. This underscores the fact that SDF is a reliable protocol in treatment of caries not only in community-based programs but also in the clinic.

With respect to secondary outcome assessment, this study observed slight tooth sensitivity in both the SDF group and the control group. However, tooth sensitivity was found to be statistically significant in the control group at 1 month and 3 months' 
periods of assessment $(p<0.05)$. The significant tooth sensitivity in the control group could likely be due to the fact that the study was field-based as against clinic based and caries excavation before placement of GIC may not have been very thorough. Secondly radiographic evaluation of carious teeth was not done in the field. Nevertheless, Milgrom et al. ${ }^{19}$ in a placebo controlled trial reported $16.0 \%$ slight tooth sensitivity in the SDF group even though it was not significant.

This study observed more intervention failures in the control group compared to the study group. The study recorded $47.5 \%$ failure rate in the control group and total loss of restoration accounted for the highest proportion (40.8\%). The treatment of multiple participants on the same day on the field rather than in the clinic where improved moisture control, superior lighting source and radiographic evaluation is more feasible may likely have contributed to this high failure rate. More importantly however, it has been documented that the drawbacks of GIC include inadequate flexural strength, little toughness and low abrasive resistance leading to wear and loss of restoration thus necessitating frequent recall visits and follow up ${ }^{34}$. Nevertheless similar studies have also recorded this proportion of failure in Atraumatic Restorative Treatment (ART) control group treatments ${ }^{17,38}$.

Many studies have documented and reported black staining of carious lesions as significant adverse effect associated with SDF treatment ${ }^{14,16,29,32,33}$. In this study, black staining of carious lesions was also observed to be associated with SDF treatment, which was significant at different assessment intervals. Some studies recommend the use of potassium iodide along with SDF to keep staining to a minimum. No other adverse effect was observed to be significant in the SDF group. According to FDA, tooth discoloration is not considered as harm that causes damage to health. However, this effect cannot be totally ignored as this drawback may cause dissatisfaction for children and parents, especially when treating anterior teeth. In addition, it can result in skin and mucosal stains which disappears after 2 days, thus, caution should be taken when applying this medicament.

This study however has some limitations. Paucity of published work on this subject in this part of the world made it difficult to have a sufficiently extensive local comparison for the findings from the study. Furthermore, the assessment outcome of the study, which was proposed to be in 2 weeks, 1 month, 3 months and 6 months respectively, was limited to 2 weeks, 1 month and 3 months due to the shutdown of schools for many months in 2020 occasioned by the COVID 19 pandemic. However, being the first study in Nigeria and possibly the whole of Africa to evaluate the effectiveness of 38\% Silver Diamine Fluoride in arresting caries, it provides vital reference data for further studies and also a possible template for policy proposals for implementing field based secondary preventive initiatives among Nigeria's large population of indigent children who have limited access to dental care.

In conclusion, the result of the study showed that SDF was effective in arresting caries in children without any harm and there was statistically significant difference in the use of $38 \%$ SDF in arresting caries in children. The mean \pm SD and Confidence Interval $(\mathrm{CI})$ of arrested caries in the SDF group were $113 \pm 1.24$ and 113.1 - 113.5 respectively. In the control group the mean \pm SD and Cl of restor- 
ative success were $69.3 \pm 11.8$ and $67.2-71.4$. The effect size ' $d$ ' of the study was 5.24. SDF was demonstrated to be effective and safe for short-term treatment of dental caries in children.

\section{Source of Funding}

This research did not receive any specific grant from funding agencies in the public, commercial, or not for- profit sectors.

\section{Conflict of interest}

There are no conflicts of interest with respect to the study.

\section{Dataset availability}

Datasets related to this article will be available upon request to the corresponding author.

\section{References}

1. GBD 2016 Disease and Injury Incidence and Prevalence Collaborators. Global, regional, and national incidence, prevalence, and years lived with disability for 328 diseases and injuries for 195 countries, 1990-2016: a systematic analysis for the Global Burden of Disease Study 2016. Lancet. 2017 Sep;390(10100):1211-59. doi: 10.1016/S0140-6736(17)32154-2.

2. Adeniyi AA, Oyapero OA, Ekekezie OO, Braimoh MO. Dental caries and nutritional status of School Children In Lagos, Nigeria - a preliminary survey. J West Afr Coll Surg. 2016 Jul-Sep;6(3):15-38.

3. Oyapero A, Bakare TI, Goncalves TF. Advancing oral health policy for mandatory dental screening before admission into public primary and secondary schools in Lagos, Nigeria. J Family Med Prim Care. 2020 Dec 31;9(12):5988-94. doi: 10.4103/jfmpc.jfmpc_1341_20.

4. Adeniyi AA, Agbaje O, Onigbinde O, Ashiwaju O, Ogunbanjo O, Orebanjo O, et al. Prevalence and pattern of dental caries among a sample of nigerian public primary school children. Oral Health Prev Dent. 2012;10(3):267-74.

5. Centres for Disease Control and Prevention. U.S. Department of Health and Human Services. Office of Disease Prevention and Health Promotion. Office of the Assistant Secretary for Health, Office of the Secretary. Healthy People 2010. [cited 21 Dec 2013]. Available from: http://www.healthypeople.gov.

6. Centres for Disease Control and Prevention. National Center for Health Statistics. Healthy People 2010 Final Review. Hyattsville, MD; 2012. Available from: https://www.cdc.gov/nchs/healthy_people/ hp2010/hp2010_final_review.htm.

7. Costa SM, Martins CC, Bonfim ML, Zina LG, Paiva SM, Pordeus IA, et al. A systematic review of socioeconomic indicators and dental caries in adults. Int J Environ Res Public Health. 2012 Oct 10;9(10):3540-74. doi: 10.3390/ijerph9103540.

8. Charles JM. Dental care in children with developmental disabilities: attention deficit disorder, intellectual disabilities, and autism. J Dent Child (Chic). 2010 May-Aug;77(2):84-91.

9. Subcommittee on Attention-Deficit/Hyperactivity Disorder; Steering Committee on Quality Improvement and Management, Wolraich M, Brown L, Brown RT, DuPaul G, Earls M, et al. ADHD: clinical practice guideline for the diagnosis, evaluation, and treatment of attentiondeficit/hyperactivity disorder in children and adolescents. Pediatrics. 2011 Nov;128(5):1007-22. doi: 10.1542/peds.2011-2654. 
10. Slayton RL, Urquhart O, Araujo MWB, Fontana M, Guzmán-Armstrong S, Nascimento MM, et al. Evidence-based clinical practice guideline on nonrestorative treatments for carious lesions: a report from the American Dental Association. J Am Dent Assoc. 2018 Oct;149(10):837-49.e19. doi: 10.1016/j.adaj.2018.07.002.

11. Peng JJ, Botelho MG, Matinlinna JP. Silver compounds used in dentistry for caries management: a review. J Dent. 2012 Jul;40(7):531-41. doi: 10.1016/j.jdent.2012.03.009.

12. Barker C. The Lund Report. Breakthrough Therapy Designation for Silver Diamine Fluoride granted by FDA; 2016 Nov [cited 2019 Jan 12]. Available from: https://www.thelundreport.org/content/ breakthrough-therapy-designation-silver-diamine-fluoride-granted-fda.

13. Tan HP, Lo EC, Dyson JE, Luo Y, Corbet EF. A randomized trial on root caries prevention in elders. J Dent Res. 2010 Oct;89(10):1086-90. doi: 10.1177/0022034510375825.

14. Rosenblatt A, Stamford TC, Niederman R. Silver diamine fluoride: a caries "silver-fluoride bullet". J Dent Res. 2009 Feb;88(2):116-25. doi: 10.1177/0022034508329406.

15. Chu CH, Lo EC. Promoting caries arrest in children with silver diamine fluoride: a review. Oral Health Prev Dent. 2008;6(4):315-21.

16. Yee R, Holmgren C, Mulder J, Lama D, Walker D, van Palenstein Helderman W. Efficacy of silver diamine fluoride for Arresting Caries Treatment. J Dent Res. 2009 Jul;88(7):644-7. doi: $10.1177 / 0022034509338671$.

17. Zhi QH, Lo EC, Lin HC. Randomized clinical trial on effectiveness of silver diamine fluoride and glass ionomer in arresting dentine caries in preschool children. J Dent. 2012 Nov;40(11):962-7. doi: 10.1016/j.jdent.2012.08.002.

18. Mei ML, Ito L, Cao Y, Lo EC, Li QL, Chu CH. An ex vivo study of arrested primary teeth caries with silver diamine fluoride therapy. J Dent. 2014 Apr;42(4):395-402. doi: 10.1016/j.jdent.2013.12.007.

19. Milgrom P, Horst JA, Ludwig S, Rothen M, Chaffee BW, Lyalina S, et al. Topical silver diamine fluoride for dental caries arrest in preschool children: A randomized controlled trial and microbiological analysis of caries associated microbes and resistance gene expression. J Dent. 2018 Jan;68:72-8. doi: 10.1016/j.jdent.2017.08.015.

20. Wikipedia. Lagos State. Lagos State Demographs. 2015 Oct 18 [ cited 2016 Feb 21]. Available from: https://en.m.wikipedia.org>wiki>Lagos.

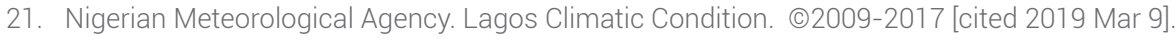
Available from: www.lagos.climatemps.com>humidity.

22. National Sugar Development Council. Report of the National Wide Survey on Industrial Sugar Consumption in Nigeria; 2014 [cited 2015 Aug 16]. Available from: http://www.nsdcnigeria.org/ wp-content/uploads/2016/07/2014-REPORT-ON-ANNUAL-SURVEY-ON-INDUSTRIAL-SUGARCONSUMPTION-IN-NIGERIA.pdf

23. Mei ML, Chu CH, Lo EC, Samaranayake LP. Fluoride and silver concentrations of silver diammine fluoride solutions for dental use. Int J Paediatr Dent. 2013 Jul;23(4):279-85. doi: 10.1111/ipd.12005.

24. Pitts N. "ICDAS"--an international system for caries detection and assessment being developed to facilitate caries epidemiology, research and appropriate clinical management. Community Dent Health. 2004 Sep;21(3):193-8.

25. Tedequim Products. "Silver Diamine Fluoride - A caries Arrest Agent" [cited 2019 Feb 11. Available from: https://www.tedequim.com.

26. Prevest Denpro. Glass ionomer restorative self-cure in hand mix version for restoration of primary teeth core build up [cited 2019 Jul 22]. Available from: http://www.prevestdenpro.com.

27. Sarvas E, KarpJM. Silver diamine fluoride arrests untreated dental caries but has drawbacks. AAP News J. 2016 Aug 5 [cited 2021 Mar 11]. Available from: https://www.aappublications.org/ news/aapnewsmag/2016/08/05/SilverDiamine080516.full-text.pdf. 
28. Contreras V, Toro MJ, Elías-Boneta AR, Encarnación-Burgos A. Effectiveness of silver diamine fluoride in caries prevention and arrest: a systematic literature review. Gen Dent. 2017 May-Jun;65(3):22-9 [cited 2021 Mar 11]. Available from: https://www.agd.org/docs/defaultsource/self-instruction-(gendent)/gendent_mj17_contreras.pdf.

29. Chu CH, Lo EC, Lin HC. Effectiveness of silver diamine fluoride and sodium fluoride varnish in arresting dentin caries in Chinese pre-school children. J Dent Res. 2002 Nov;81(11):767-70. doi: $10.1177 / 0810767$.

30. Horst JA, Ellenikiotis H, UCSF Silver Caries Arrest Committee, Milgrom PM. UCSF protocol for caries arrest using silver diamine fluoride: rationale, indications, and consent. J Calif Dent Assoc. 2016 Jan;44(1):16-28.

31. Alvear BA, Horst JA, Hirsch JP, Duffin S, Wong A, Young DA. Arresting caries. Dimens Dent Hyg. 2016 Jul; 14(7):61-4.

32. Clemens J, Gold J, Chaffin J. Effect and acceptance of silver diamine fluoride treatment on dental caries in primary teeth. J Public Health Dent. 2018 Dec;78(1):63-68. doi: 10.1111/jphd.12241.

33. Llodra JC, Rodriguez A, Ferrer B, Menardia V, Ramos T, Morato M. Efficacy of silver diamine fluoride for caries reduction in primary teeth and first permanent molars of schoolchildren: 36-month clinical trial. J Dent Res. 2005 Aug;84(8):721-4. doi: 10.1177/154405910508400807.

34. Nishino M. [Studies on the topical application of ammoniacal silver fluoride for the arrest of dental caries]. Osaka Daigaku Shigaku Zasshi. 1969 Jun;14(1):1-14. Japanese.

35. Li R, Lo EC, Liu BY, Wong MC, Chu CH. Randomized clinical trial on arresting dental root caries through silver diammine fluoride applications in community-dwelling elders. J Dent. 2016 Aug;51:15-20. doi: 10.1016/j.jdent.2016.05.005.

36. Ahovuo-Saloranta A, Forss H, Walsh T, Hiiri A, Nordblad A, Mäkelä M, Worthington HV. Sealants for preventing dental decay in the permanent teeth. Cochrane Database Syst Rev. 2013 Mar 28;(3):CD001830. doi: 10.1002/14651858.CD001830.pub4. Update in: Cochrane Database Syst Rev. 2017 Jul 31;7:CD001830.

37. Santos Jr VE, Vasconcelos Filho A, Targino AG, Flores MA, Galembeck A, Caldas AF Jr, et al. A new "silver-bullet" to treat caries in children--nano silver fluoride: a randomised clinical trial. J Dent. 2014 Aug;42(8):945-51. doi: 10.1016/j.jdent.2014.05.017.

38. Gao SS, Zhao IS, Hiraishi N, Duangthip D, Mei ML, Lo ECM, et al. Clinical trials of Silver Diamine Fluoride in arresting caries among children: a systematic review. JDR Clin Trans Res. 2016 Oct; 1(3):201-10. doi: 10.1177/2380084416661474.

39. Monse B, Heinrich-Weltzien R, Mulder J, Holmgren C, van Palenstein Helderman WH. Caries preventive efficacy of silver diammine fluoride (SDF) and ART sealants in a school-based daily fluoride toothbrushing program in the Philippines. BMC Oral Health. 2012 Nov 21;12:52. doi: 10.1186/1472-6831-12-52.

40. Dos Santos VE Jr, de Vasconcelos FM, Ribeiro AG, Rosenblatt A. Paradigm shift in the effective treatment of caries in schoolchildren at risk. Int Dent J. 2012 Feb;62(1):47-51. doi: 10.1111/j.1875-595X.2011.00088.x.

41. Braga MM, Mendes FM, De Benedetto MS, Imparato JC. Effect of silver diammine fluoride on incipient caries lesions in erupting permanent first molars: a pilot study. J Dent Child (Chic). 2009 Jan-Apr;76(1):28-33.

42. Castillo JL, Rivera S, Aparicio T, Lazo R, Aw TC, Mancl LL, Milgrom P. The short-term effects of diammine silver fluoride on tooth sensitivity: a randomized controlled trial. J Dent Res. 2011 Feb;90(2):203-8. doi: 10.1177/0022034510388516. 